\title{
Mechanism of decoherence-free coupling between giant atoms
}

\author{
Angelo Carollo $\odot,{ }^{1,2}$ Dario Cilluffo, ${ }^{1,3}$ and Francesco Ciccarello ${ }^{1,3}$ \\ ${ }^{1}$ Università degli Studi di Palermo, Dipartimento di Fisica e Chimica-Emilio Segrè, via Archirafi 36, I-90123 Palermo, Italy \\ ${ }^{2}$ Radiophysics Department, National Research Lobachevsky State University of Nizhni Novgorod, 23 Gagarin Avenue, \\ Nizhni Novgorod 603950, Russia \\ ${ }^{3}$ NEST, Istituto Nanoscienze-CNR, Piazza S. Silvestro 12, 56127 Pisa, Italy
}

(Received 27 June 2020; accepted 22 September 2020; published 4 November 2020)

\begin{abstract}
Giant atoms are a new paradigm of quantum optics going beyond the usual local coupling. Building on this, a new type of decoherence-free (DF) many-body Hamiltonians was shown in a broadband waveguide. Here these are incorporated in a general framework (not relying on master equations) and contrasted to dispersive DF Hamiltonians with normal atoms: the two schemes are shown to correspond to qualitatively different ways to match the same general condition for suppressing decoherence. Next, we map the giant atoms dynamics into a cascaded collision model (CM), providing an intuitive interpretation of the connection between nontrivial DF Hamiltonians and coupling points topology. The braided configuration is shown to implement a scheme where a shuttling system subject to periodic phase kicks mediates a DF coupling between the atoms. From the viewpoint of CMs theory, this shows a collision model where ancillas effectively implement a dissipationless, maximally entangling two-qubit gate on the system.
\end{abstract}

DOI: 10.1103/PhysRevResearch.2.043184

\section{INTRODUCTION}

Engineering decoherence-free (DF) Hamiltonians is a major task in the field of quantum technologies and many-body physics, with special regard to quantum optics implementations [1-7]. In particular, DF mediated Hamiltonians describe coherent interactions, typically between (pseudo) atoms or qubits which crosstalk via a quantum bus (usually some photonic environment) that yet does not introduce decoherence [8]. In terms of the Lindblad master equation [9], this implies realizing a net second-order Hamiltonian that couple the atoms to one another, getting rid at once of the (usually present) dissipator term. Thereby one is left with an effective unitary dynamics of the atoms, where the environmental degrees of freedom are eliminated.

One of the typical strategies to achieve DF Hamiltonians is adiabatic elimination in the dispersive regime. In cavity QED, it is typically obtained by coupling a set of atoms far off-resonantly to cavity modes [10-12]. This gives rise to a separation of timescales such that incoherent second-order interactions average to zero, while coherent ones result in an effective Hamiltonian. An analogous working principle underpins DF Hamiltonians in structured photonic lattices [5,6,13], which are seeded by tuning the atomic frequency within a photonic band gap entailing an off-resonant interaction with

Published by the American Physical Society under the terms of the Creative Commons Attribution 4.0 International license. Further distribution of this work must maintain attribution to the author(s) and the published article's title, journal citation, and DOI. all the lattice modes (this results in short-range, potentially tunable, interatomic couplings).

Recently, a new class of DF Hamiltonians was predicted [14] and experimentally observed [15], which employs giant atoms [16] in broadband waveguides. Giant atoms are a new playground of quantum optics [17-23], where the usual pointlike model of an emitter (normal atom) breaks down. In contrast, as sketched in Fig. 1, a giant atom (typically an artificial two-level system) couples to the field at a discrete set of distinct coupling points (an alternative implementation is an atom in front of a mirror [24-26]). By appropriate engineering, the distance between coupling points can be made several wavelengths long. This introduces tunable phase shifts, yielding interference effects unattainable with normal atoms. Notably, these can be harnessed in particular to suppress dissipative interactions, giving rise to DF Hamiltonians [14,19]. As a distinctive feature of giant atoms, such Hamiltonians can happen to be trivial (i.e., identically zero or with null coupling terms) depending on the coupling points topology. For two giant atoms, for instance, only one out of the three possible topologies leads to nontrivial DF Hamiltonians [14,15].

So far occurrence of giant atoms DF Hamiltonians was mostly investigated through the explicit master equation of the atoms [14] derived via the SLH formalism [27], in turn related to the input-output theory for waveguide-QED setups [28]. This master equation is a rather involved object featuring both local and nonlocal dissipative terms, based on which the essential mechanism behind emergence of DF Hamiltonians is not straightforwardly interpreted. In the case of the aforementioned DF dispersive Hamiltonians, however, a derivation solely based on average Hamiltonian theory [29-33] (thus not relying on master equations) is possible [12]. One thus 


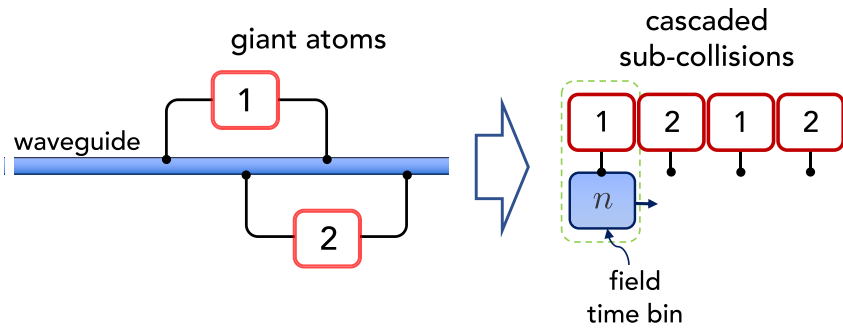

FIG. 1. Two giant atoms, each coupled to a waveguide at two coupling points. The dynamics is mapped into cascaded subcollisions, each involving one field time bin and one coupling point.

naturally asks whether a similar picture can be defined for giant atoms and used to study conditions for occurrence of DF Hamiltonians.

With the above motivations, in this work we consider a general framework for deriving DF Hamiltonians in the spirit of average Hamiltonian theory. The main condition ("DF condition") is to arrange for an interaction Hamiltonian averaging to zero over a coarse-grained timescale (in the interaction picture). Dispersive schemes and giant atoms in a broadband waveguide are compared and shown to be different ways to match the DF condition. This occurs through destructive interference of a continuum of phase factors defining the interaction Hamiltonian in the former case and only a discrete, possibly small, number in the latter. We next focus on giant atoms schemes, reviewing their general description through a collision-model picture and connecting it to the DFHamiltonians framework. It is then shown that each collective collision with all the atoms can be decomposed as a cascade of ordered subcollisions (see Fig. 1), each involving a single coupling point. Topologies yielding a zero effective Hamiltonian (such as serial and nested configurations) correspond to subcollisions combined with their time-reversed analogs so as to produce a zero net evolution. Instead, nonzero DF Hamiltonians are seeded for topologies (such as the braided) that feature combinations of subcollisions and their time-reversed versions not leading to an overall identity evolution.

Moreover, we show that the cascaded-collision picture allows us to map giant atoms dynamics into a mediator (embodied by a field time bin) shuttling between the atoms and subject to periodic phase kicks. The equivalent quantum circuit, a sequence of parametric iSWAP and local phase gates, is presented.

This paper starts in Sec. II by developing a general framework for occurrence of DF Hamiltonians based on the Magnus expansion of the joint propagator in each time interval. We next review in Sec. III how the necessary requirements for having a DF Hamiltonian are fulfilled in the case of standard dispersive Hamiltonians: this provides an illustration of the theory of Sec. II in a familiar setup with which DF Hamiltonians via giant atoms (our main focus) will be profitably compared. In Sec. IV giants atoms coupled to a broadband waveguide are introduced, showing how they provide a different way to match the DF condition compared to dispersive schemes. In Sec. V we review how the joint dynamics of giant atoms and field can be described through an average-Hamiltonian approach, decomposing into a sequence of elementary unitaries in each of which the atoms jointly collide with a field time bin. Section VI shows how each collision in turn can be decomposed into cascaded subcollisions, one for each coupling point. This highlights the physical origin of the effective Hamiltonian so as to link it to the coupling points topology, a task carried out in Sec. VII. In Sec. VIII we show that the giant-atoms setup can be seen as an implementation of a scheme where a shuttling qubit, subject to periodic phase gates, mediates an indirect DF coupling between the atoms, and the equivalent quantum circuit is given. The theory developed in Secs. VI and VII is extended to a bidirectional (generally chiral) waveguide in Sec. IX, and to more than two coupling points in Sec. X. Finally, we draw our conclusions in Sec. XI.

\section{GENERAL SCHEME FOR DECOHERENCE-FREE HAMILTONIANS}

Consider an unspecified quantum system $S$ coupled to a quantum environment $E$. The Hamiltonian reads

$$
H=H_{S}+H_{E}+V,
$$

with $H_{S}\left(H_{E}\right)$ the free Hamiltonian of $S(E)$ and $V$ their interaction Hamiltonian. In the interaction picture with respect to $H_{0}=H_{S}+H_{E}$, the joint state $\sigma_{t}$ evolves as

$$
\dot{\sigma}_{t}=-i\left[V_{t}, \sigma_{t}\right],
$$

with $V_{t}=e^{i H_{0} t} V e^{-i H_{0} t}$. Thus at time $t$,

$$
\sigma_{t}=\mathcal{U}_{t} \sigma_{0} \mathcal{U}_{t}^{\dagger},
$$

with the propagator $\mathcal{U}_{t}$ given by $\mathcal{U}_{t}=\mathcal{T} \exp \left[-i \int_{t_{0}}^{t} d s V(s)\right]$, where $\mathcal{T}$ is the usual time ordering operator.

Consider now a mesh of the time axis defined by $t_{n}=n \Delta t$ with $n=0,1, \ldots$ and $\Delta t$ the time step, in terms of which the propagator can be decomposed as

$$
\mathcal{U}_{t}=\prod_{n=1}^{[t / \Delta t]} U_{n}, \quad \text { with } \quad U_{n}=\mathcal{T} e^{-i \int_{t_{n-1}}^{t_{n}} d s V(s)} .
$$

Now, if $\Delta t$ is short enough compared to the characteristic time of interaction, applying the Magnus expansion [34] each unitary $U_{n}$ can be approximated to second order as

$$
U_{n} \simeq \mathbb{1}-i\left(\bar{V}_{n}+\mathcal{H}_{n}\right) \Delta t-\frac{1}{2} \bar{V}_{n}^{2} \Delta t^{2},
$$

with $\mathbb{1}$ the identity operator and

$$
\begin{gathered}
\bar{V}_{n}=\frac{1}{\Delta t} \int_{t_{n-1}}^{t_{n}} d s V_{s}, \\
\mathcal{H}_{n}=-\frac{i}{2 \Delta t} \int_{t_{n-1}}^{t_{n}} d s \int_{t_{n-1}}^{s} d s^{\prime}\left[V_{s}, V_{s^{\prime}}\right] .
\end{gathered}
$$

The averaged interaction $\bar{V}_{n}$ and Hamiltonian $\mathcal{H}_{n}$, respectively, of first and second order in the coupling strength, are the two central quantities to consider for implementing DF Hamiltonians. In sketchy terms, one seeks to fulfill $\bar{V}_{n}=0$ (henceforth referred to as the "DF condition") in a way that $\mathcal{H}_{n}$ yields (upon partial trace) a dissipationless effective Hamiltonian of $S, H_{\text {eff }}$. This is formalized in detail in the following. 
Let $\sigma_{n}$ be the joint $S$-E state at time $t_{n}$ and $\rho_{n}=\operatorname{Tr}_{E}\left\{\sigma_{n}\right\}$ the reduced state of the system at the same time. We will consider a coarse-grained timescale defined by $\Delta t$ short enough that (5) holds. In the corresponding continuous-time limit, $t_{n} \rightarrow t, \sigma_{n} \rightarrow \sigma_{t}, \Delta \sigma_{n} / \Delta t \rightarrow \dot{\sigma}$, where we set $\Delta \sigma_{n}=\sigma_{n}-$ $\sigma_{n-1}$ (analogously for $\rho_{n}$ ).

We also define

$$
\begin{gathered}
\left\langle\mathcal{H}_{n}\right\rangle_{\rho_{0}}=\operatorname{Tr}_{S}\left\{\mathcal{H}_{n} \rho_{0} \otimes \mathbb{1}_{E}\right\}, \\
H_{\text {eff }}=\operatorname{Tr}_{E}\left\{\mathcal{H}_{n} \mathbb{1}_{S} \otimes \rho_{E}\right\},
\end{gathered}
$$

with $\operatorname{Tr}_{S(E)}\{\}$ the partial trace over $S(E)$. These are effective Hamiltonians on $E$ and $S$, respectively. When $S$ is multipartite, in particular, $H_{\text {eff }}$ will generally feature mutual couplings between subsystems of $S$.

The following property holds.

Property. Let the system and environment be initially in the uncorrelated state $\rho_{0} \otimes \rho_{E}$ with $\rho_{0}\left(\rho_{E}\right)$ the initial state of the system (environment). If

$$
\bar{V}_{n}=0
$$

in each time interval $\left[t_{n-1}, t_{n}\right]$, and

$$
\left[\mathcal{H}_{n}, \mathbb{1}_{S} \otimes \rho_{E}\right]=0,
$$

then in the continuous-time limit

$$
\dot{\rho}=-i\left[H_{\mathrm{eff}}, \rho\right] .
$$

This embodies a rather general working principle for realizing DF effective Hamiltonians: conditions (10) and (11) entail a unitary reduced dynamics of $S$ generated by the effective Hamiltonian $H_{\text {eff }}$. Among (10) and (11), the former (DF condition) is the most relevant: it means that the interaction Hamiltonian $V_{t}$ averages to zero over the coarse-grained timescale $\Delta t$

The above property is easily shown (see Appendix A), from which in particular it turns out that $\sigma_{n}=\rho_{n} \otimes \rho_{E}$, namely $E$ remains in its initial state, uncorrelated with $S$.

A typical case where (11) occurs is when $\mathcal{H}_{n}$ acts trivially on $E$, then (11) is matched for any $\rho_{E}$ and $\mathcal{H}_{n} \equiv H_{\text {eff }}$ (this happens with giant atoms as we will see). Another instance is when $S$ is a two-level system and $E$ is a harmonic oscillator with $\mathcal{H}_{n} \sim \sigma_{z} b^{\dagger} b$ (dispersive regime of the Jaynes-Cummings model [35]; see next section). Then (11) holds when $\rho_{E}$ is any mixture of Fock states.

Note that the above framework, alongside related approaches $[31,32,36]$, bypasses any direct use of master equations or the Born-Markov approximation, being instead mostly based on propagators and Hamiltonians.

\section{DISPERSIVE HAMILTONIANS}

A longstanding way for matching condition (10) in quantum optics is coupling atoms to a single- or multimode photonic environment dispersively, i.e., off-resonantly. A standard model to illustrate this is a set of identical two-level atoms of frequency $\omega_{0}$ and ground (excited) state $|g\rangle(|e\rangle)$ weakly coupled to a bosonic field. The atoms play the role of system $S$ and the field of environment $E$. Their free Hamil- tonians read

$$
H_{S}=\omega_{0} \sum_{j} \sigma_{j}^{\dagger} \sigma_{j}, \quad H_{E}=\sum_{k} \omega_{k} b_{k}^{\dagger} b_{k},
$$

while the interaction Hamiltonian in the rotating-wave approximation is given by

$$
V=\sum_{j, k} g_{j k} \sigma_{j} b_{k}^{\dagger}+\text { H.c. },
$$

with coupling strength $g_{j k}$ generally complex, $\sigma_{j}=|g\rangle_{j}\langle e|$ and $b_{k}$ bosonic ladder operators of the field (here $k$ labels the field normal modes and in general could comprise both discrete and continuous indexes).

In the interaction picture, $V$ turns into

$$
V_{t}=\sum_{j} \sum_{k} g_{j k} \sigma_{j} b_{k}^{\dagger} e^{i \Delta_{k} t}+\text { H.c. },
$$

with $\Delta_{k}=\omega_{k}-\omega_{0}$ the detuning between mode $k$ and the atomic transition frequency $\omega_{0}$.

Consider now the off-resonance regime such that the detunings are all much larger than the typical order of magnitude of the interaction, which is generally expressed as $\min _{k}\left|\Delta_{k}\right| \gg$ $\max _{j k}\left|g_{j k}\right|$. Then one can choose a coarse-grained timescale $\Delta t$ such that

$$
\max _{k}\left|\Delta_{k}\right|^{-1} \ll \Delta t \ll \min _{j k}\left|g_{j k}\right|^{-1} .
$$

Accordingly,

$$
\int_{t_{n-1}}^{t_{n}} d t e^{ \pm i \Delta_{k} t} \simeq 0 \text { for any } k,
$$

hence (15) averages to zero in each time interval $\left[t_{n-1}, t_{n}\right]$ of length $\Delta t$ so as to fulfill the DF condition (10). The Hamiltonian term (7) is given by

$$
\begin{aligned}
\mathcal{H}_{n}= & -\sum_{j, j^{\prime}} \sum_{k} \frac{g_{j, k} g_{j^{\prime}, k}^{*}}{2 \Delta_{k}} \sigma_{j^{\prime}}^{\dagger} \sigma_{j}+\text { H.c. } \\
& +\sum_{j} \sum_{k, k^{\prime}}^{\prime} \frac{g_{j, k} g_{j, k^{\prime}}^{*}}{\Delta_{k}} \sigma_{j z} b_{k}^{\dagger} b_{k^{\prime}},
\end{aligned}
$$

with the primed sum running over all $k, k^{\prime}$ such that $\omega_{k}=\omega_{k^{\prime}}$. For a single atom and only one field mode, this reduces to the interaction Hamiltonian $\sim \sigma_{z} b^{\dagger} b$ arising in the dispersive regime of the Jaynes-Cummings model [37].

Choosing $\rho_{E}=|0\rangle\langle 0|$, with $|0\rangle$ the field vacuum state, condition (11) is fulfilled, hence we get the DF effective Hamiltonian [cf. Eq. (9)]

$$
H_{\mathrm{eff}}=-\sum_{j, j^{\prime}} \sum_{k} \frac{g_{j, k} g_{j^{\prime}, k}^{*}}{2 \Delta_{k}} \sigma_{j^{\prime}}^{\dagger} \sigma_{j}+\text { H.c. }
$$

featuring atom-atom couplings.

\section{GIANT ATOMS IN A BROADBAND WAVEGUIDE}

A standard way to realize the scheme in the previous section is to couple the atoms to a photonic lattice and tune $\omega_{0}$ far from any band (see Fig. 2). The atoms then interact with the photonic environment far off-resonantly, which results in the separation of timescales (16). 


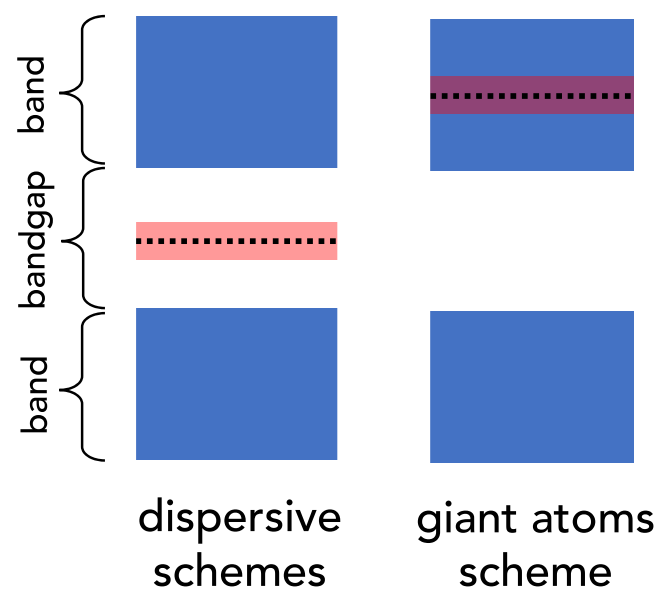

FIG. 2. Frequency regimes for realizing DF Hamiltonians. The photonic environment typically features frequency bands separated by band gaps. The dashed line marks the atomic frequency $\omega_{0}$, which couples resonantly with a bandwidth of modes (in red) of the order of the atom-field coupling rate. Dispersive schemes (left panel) work off-resonance by tuning atoms far off-resonance from lattice bands. Instead, DF Hamiltonians with giant atoms (on the right) operate well within a photonic band, which can thus be approximated as infinite.

Instead, decoherence-free Hamiltonians via giant atoms work in the regime in which the atomic frequency $\omega_{0}$ is well within a photonic band which can thus be approximated as infinite (see Fig. 2). This is possible due to nonlocal coupling (the hallmark of giant atoms) as will become clear later. Thus consider a set of giant two-level atoms weakly coupled to a one-dimensional waveguide [38-40] with $\omega_{0}$ inside a band of the waveguide field. The free atomic Hamiltonians of $S$ and $E$ are still given by Eq. (13), where (compared to the general case in the previous section) $k$ is now intended as the wave vector. The $j$ th atom is coupled to the waveguide at $\mathcal{N}_{j}$ distinct coupling points [see Fig. 3(a)], the coordinate of each being $x_{j \ell}$ with $\ell=1, \ldots, \mathcal{N}_{j}$ (such that $x_{j 1}<x_{j 2}<\cdots$ ). Accordingly, the interaction Hamiltonian in the interaction picture now reads

$$
V_{t}=\sum_{j, \ell} \sum_{k} g_{j \ell k} \sigma_{j} b_{k}^{\dagger} e^{i \Delta_{k} t}+\text { H.c. }
$$

with $g_{j \ell k}$ the coupling strength to mode $k$ of the $\ell$ th coupling point of atom $j$. Unlike the previous section, resonant modes $k \simeq \pm k_{0}$ (with $\omega_{k_{0}}=\omega_{0}$ ) will now dominate, thus (17) does not hold.

The coupling strengths more explicitly read $g_{j \ell k}=$ $g_{k} e^{-i k x_{j \ell}}$, where taking advantage of weak coupling we can approximate $g_{k} \simeq g_{k_{0}}$ (which thus become $k$ independent). Accordingly, we can write

$$
g_{j \ell k}=g_{k_{0}} e^{-i k_{0} x_{j \ell}} e^{-i\left(k-k_{0}\right) x_{j \ell}},
$$

where (for the sake of argument) we are assuming for now a unidirectional field. Plugging into $V_{t}$, we get

$$
V_{t}=g_{k_{0}} \sum_{j, \ell} e^{-i \varphi_{j \ell}} \sigma_{j} \sum_{k} e^{-i\left(\Delta_{k} t-k x_{j \ell}\right)} b_{k}^{\dagger}+\text { H.c. }
$$

(a)

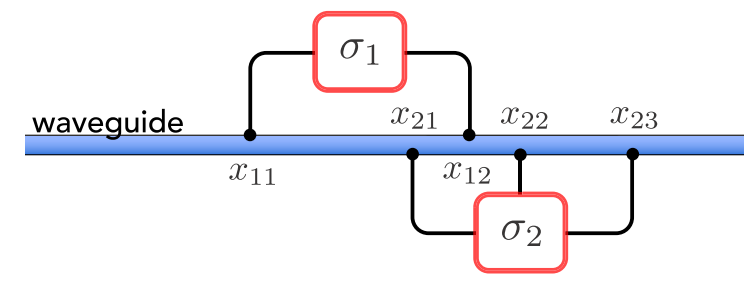

(b)

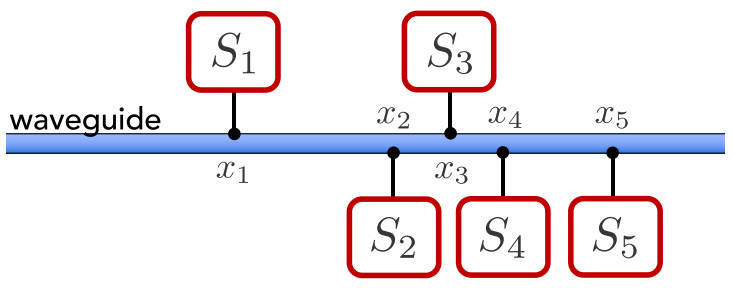

FIG. 3. Giant atoms coupled to a waveguide. (a) Instance with two atoms ( 1 and 2) having $\mathcal{N}_{1}=2$ and $\mathcal{N}_{2}=3$ coupling points, each of coordinate $x_{j \ell}$ with $x_{j 1}<x_{j 2}<\cdots$. (b) For each coupling point $v=1, \ldots, \mathcal{N}$ ( $v$ growing from left to right) we define an atomic operator $S_{v}$ [cf. Eq. (27)] as the $\sigma_{j}$ of the corresponding atom times the coupling-point phase factor $e^{-i \varphi_{j \ell}}$. For example, $S_{1}=\sigma_{1} e^{-i \varphi_{11}}, S_{4}=\sigma_{2} e^{-i \varphi_{22}}$ with $\varphi_{j \ell}=k_{0} x_{j \ell}$. Note that $S_{v}$ generally does not commute with $S_{v^{\prime} \neq v}^{\dagger}$. Atomic operators $S_{v}^{\prime}$ are defined analogously, except that the phase changes sign, $\varphi_{j \ell} \rightarrow-\varphi_{j \ell}$.

where we defined the coupling point phases

$$
\varphi_{j \ell}=k_{0} x_{j \ell}
$$

and performed the variable change $k \rightarrow k-k_{0}$ (wave vector measured from $k_{0}$ ). Consistently with the weak-coupling regime, we can linearize the photonic dispersion law around the atomic frequency as $\omega_{k} \simeq \omega_{0}+v k$ with $v$ the photon velocity. Using $v$, the coupling points coordinates can be expressed in the time domain as $\tau_{j \ell}=x_{j \ell} / v$. Thereby, (21) becomes

$$
V_{t}=g_{k_{0}} \sum_{j, \ell} e^{-i \varphi_{j \ell}} \sigma_{j} \sum_{k} e^{-i \omega_{k}\left(t-\tau_{j \ell}\right)} b_{k}^{\dagger}+\text { H.c. }
$$

Averaging (23) over a time interval $\left[t_{n-1}, t_{n}\right]$ yields

$\bar{V}_{n}=g_{k_{0}} \sum_{j}\left(\sum_{\ell} e^{-i \varphi_{j \ell}}\right) \sigma_{j} \int_{t_{n-1}}^{t_{n}} d s \sum_{k} e^{-i \omega_{k}\left(s-\tau_{j \ell}\right)} b_{k}^{\dagger}+$ H.c.

(we have also split the sum over $j$ and $\ell$ ). Note that $\tau_{j^{\prime} \ell^{\prime}}-\tau_{j \ell}$ is the time delay taken by light to travel from the $\ell$ th coupling point of atom $j$ to the $\ell^{\prime}$ th coupling point of $j^{\prime}$. If all these time delays are negligible compared to $\Delta t$, then (24) can be approximated as

$$
\bar{V}_{n} \simeq g_{k_{0}} \sum_{j}\left(\sum_{\ell} e^{-i \varphi_{j \ell}}\right) \sigma_{j} \int_{t_{n-1}}^{t_{n}} d s \sum_{k} e^{-i \omega_{k} s} b_{k}^{\dagger}+\text { H.c. }
$$

Now the key point is that each atomic operator $\sigma_{j}$ comes with a prefactor $\sum_{\ell} e^{i \varphi_{j \ell}}$, which-due to nonlocal couplingcan vanish for all atoms at the same time. This occurs when the coupling point phases are adjusted so as to match 
the condition

$$
\sum_{\ell=1}^{\mathcal{N}_{j}} e^{-i \varphi_{j \ell}}=0 \quad \text { for any } j,
$$

which is the DF condition (10) for giant atoms. Note that this cannot be satisfied by normal atoms: each atom must have at least two coupling points $\left(\mathcal{N}_{j} \geqslant 2\right)$.

It is interesting to compare (26) with (17). Each can be seen as a destructive interference condition, involving a continuum of phase factors in the dispersive scheme but only a discrete, possibly small, number in the scheme with giant atoms.

\section{GIANT ATOMS DYNAMICS: AVERAGE-HAMILTONIAN DESCRIPTION}

The previous section showed how the DF condition $\bar{V}_{n}=0$ is realized with giant atoms in comparison with off-resonance schemes. When it comes to giant atoms, depending on the topology of coupling points, the DF condition can result in a vanishing (thus trivial) $H_{\text {eff }}$ [41]. Clarifying the requisites for obtaining a nontrivial effective Hamiltonian, and especially the related physical interpretation, is a major goal of this work. Prior to this, however, we reformulate the microscopic model with giant atoms in terms of time mode operators of the field (generalizing at once to a bidirectional, generally chiral, waveguide), this being the theoretical basis for the mapping of the dynamics into a cascaded collision model that will be discussed in the next sections.

\section{A. DF condition}

To begin with, as shown in Fig. 3(b), it is convenient to introduce a single index $v=1, \ldots, \mathcal{N}$ labeling all the coupling points from left to right, where $\mathcal{N}=\prod_{j} \mathcal{N}_{j}$ is the total number of coupling points. For each coupling point so indexed, we define atomic operators depending on the corresponding pair $(j, \ell)$ as

$$
S_{v}=\sigma_{j} e^{-i \varphi_{j \ell}}, \quad S_{v}^{\prime}=\sigma_{j} e^{i \varphi_{j \ell}} .
$$

For instance, in the case of Fig. 3, $S_{3}=\sigma_{1} e^{-i \varphi_{12}}$ and $S_{3}^{\prime}=$ $\sigma_{1} e^{i \varphi_{12}}$ with $\varphi_{12}=k_{0} x_{12}$ [see Eq. (22)]. Note that $S_{v}$ generally does not commute with $S_{v^{\prime} \neq v}^{\dagger}$ (e.g., in Fig. $3,\left[S_{2}, S_{5}^{\dagger}\right] \neq 0$ ).

Next, we come back to (23) and extend it to a bidirectional waveguide using the newly introduced operators as (see Ref. [42] for more details)

$$
\begin{aligned}
V_{t}= & g_{k_{0}} \sum_{\nu} S_{\nu} \sum_{k} e^{-i \omega_{k}\left(t-\tau_{v}\right)} b_{k}^{\dagger} \\
& +g_{-k_{0}} \sum_{\nu} S_{v}^{\prime} \sum_{k} e^{i \omega_{k}\left(t+\tau_{v}\right)} b_{k}^{\prime \dagger}+\text { H.c., }
\end{aligned}
$$

with ladder operators $b_{k}\left(b_{k}^{\prime}\right)$ now corresponding to rightgoing (left-going) modes [in the first (second) sum $k$ is measured from $k_{0}\left(-k_{0}\right)$ ].

In the limit in which the field becomes a continuum of modes, $V_{t}$ can be expressed in the form [42]

$$
V_{t}=\sqrt{\gamma} \sum_{\nu} S_{\nu} b_{t-\tau_{v}}^{\dagger}+\sqrt{\gamma^{\prime}} \sum_{\nu} S_{\nu}^{\prime}{b^{\prime}}_{t+\tau_{v}}^{\dagger}+\text { H.c. },
$$

with $\gamma=g_{k_{0}}^{2} / v$ and $\gamma^{\prime}=g_{-k_{0}}^{2} / v$ (which we allow to be generally different). Here $b_{t}$ are right-going time modes fulfilling $\left[b_{t}, b_{t^{\prime}}^{\dagger}\right]=\delta\left(t-t^{\prime}\right),\left[b_{t}, b_{t^{\prime}}\right]=\left[b_{t}^{\dagger}, b_{t^{\prime}}^{\dagger}\right]=0$. Likewise, $b_{t}^{\prime}$ define left-going modes with analogous commutation rules. Before proceeding further, recalling Eq. (27), it is convenient to define the collective atomic operators

$$
\begin{aligned}
& S=\sum_{\nu} S_{v}=\sum_{j}\left(\sum_{\ell} e^{-i \varphi_{j \ell}}\right) \sigma_{j}, \\
& S^{\prime}=\sum_{v} S_{v}^{\prime}=\sum_{j}\left(\sum_{\ell} e^{i \varphi_{j \ell}}\right) \sigma_{j} .
\end{aligned}
$$

The regime of negligible time delays is defined by $\tau_{\mathcal{N}}-$ $\tau_{1} \ll \gamma^{-1}, \gamma^{\prime-1}$, allowing us to coarse grain the dynamics over a characteristic timescale $\Delta t$ such that

$$
\tau_{\mathcal{N}}-\tau_{1} \ll \Delta t \ll \gamma^{-1}, \gamma^{\prime-1} .
$$

In this regime it can be shown [42] that (6) and (7) reduce to

$$
\begin{gathered}
\bar{V}_{n}=\frac{1}{\sqrt{\Delta t}}\left(\sqrt{\gamma} S b_{n}^{\dagger}+\sqrt{\gamma^{\prime}} S^{\prime} b_{n}^{\dagger}+\text { H.c. }\right), \\
\mathcal{H}_{n}=\frac{i}{2} \sum_{\nu>v^{\prime}}\left(\gamma S_{v^{\prime}}^{\dagger} S_{v}+\gamma^{\prime}{S^{\prime} \dagger}_{v^{\dagger}}^{\prime} S_{v^{\prime}}^{\prime}-\text { H.c. }\right),
\end{gathered}
$$

where

$$
b_{n}=\frac{1}{\sqrt{\Delta t}} \int_{t_{n-1}}^{t_{n}} d t b_{t}, \quad b_{n}^{\prime}=\frac{1}{\sqrt{\Delta t}} \int_{t_{n-1}}^{t_{n}} d t b_{t}^{\prime} .
$$

Equations (35) define a discrete set of ladder operators of the environment fulfilling bosonic commutation rules $\left[b_{n}, b_{n^{\prime}}^{\dagger}\right]=$ $\delta_{n, n^{\prime}},\left[b_{n}, b_{n^{\prime}}\right]=\left[b_{n}^{\dagger}, b_{n^{\prime}}^{\dagger}\right]=0$ (and likewise for $b_{n}^{\prime}$ ), as is easily checked using the commutation rules of time-mode operators $b_{t}$ and $b_{t}^{\prime}$.

Based on (33), the DF condition [cf. Eq. (10)] for a bidirectional waveguide in terms of collective atomic operators (30) and (31) simply reads

$$
S=S^{\prime}=0 .
$$

This is equivalent to (26) $\left[S=0 \Leftrightarrow S^{\prime}=0\right.$ since if Eq. (26) is matched so is the analogous equation for $\varphi_{j \ell} \rightarrow-\varphi_{j \ell}$ ].

\section{B. Effective Hamiltonian}

When $\bar{V}_{n}=0$, atoms will evolve unitarily with effective Hamiltonian [recall Eq. (9)] $\mathcal{H}_{n}=H_{\text {eff }} \otimes \mathbb{1}\left(\mathcal{H}_{n}\right.$ acts trivially on the field). Henceforth, we will omit the identity operator.

The effective Hamiltonian $H_{\text {eff }}=\mathcal{H}_{n}$ can be written more explicitly as [cf. Eqs. (27) and (34)]

$H_{\mathrm{eff}}=\frac{i}{2} \sum_{\nu>v^{\prime}}\left(\gamma e^{i\left(\varphi_{j^{\prime} \ell^{\prime}}-\varphi_{j \ell}\right)} \sigma_{j^{\prime}}^{\dagger} \sigma_{j}+\gamma^{\prime} e^{i\left(\varphi_{j^{\prime} \ell^{\prime}}-\varphi_{j \ell}\right)} \sigma_{j}^{\dagger} \sigma_{j^{\prime}}-\right.$ H.c. $)$,

where $(j, \ell)$ are understood as the pair of indexes corresponding to $v$ [and likewise $\left(j^{\prime}, \ell^{\prime}\right)$ with respect to $v^{\prime}$ ]. This in turn can be expressed in the compact form

$$
H_{\mathrm{eff}}=\sum_{j j^{\prime}} J_{j j^{\prime}} \sigma_{j}^{\dagger} \sigma_{j^{\prime}}+\text { H.c., }
$$


(a)

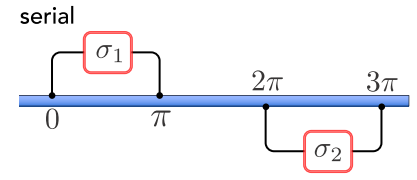

(c)

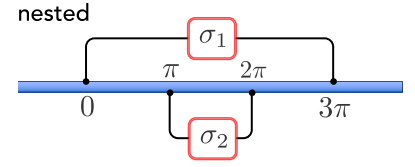

(e)

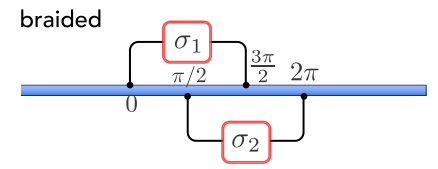

(b)

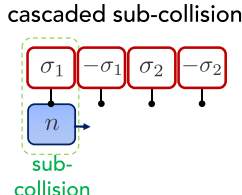

(d)

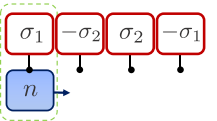

(f)

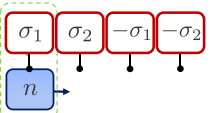

FIG. 4. Coupling point topologies of two giant atoms each with two coupling points (in a unidirectional waveguide) and corresponding sequences of cascaded subcollisions. (a) and (b) Serial topology and corresponding sequence of $S_{v}$ [cf. Eq. (27)]: $S_{1}=\sigma_{1}, S_{2}=$ $-\sigma_{1}, S_{3}=\sigma_{2}, S_{4}=-\sigma_{2}$. (c) and (d) Nested topology. (e) and (f) Braided topology. The phase $k_{0} x_{v}$ of each coupling point is shown in (a), (c), and (e). Each subcollision is described by the unitary $e^{-i V_{n v} \Delta t}$ with $V_{n v}$ given by Eq. (44). In (f) we absorbed a phase factor $e^{-i \pi / 2}$ in the definition of $\sigma_{2}$.

with

$$
\begin{aligned}
J_{j j^{\prime}}= & \sum_{v_{j^{\prime} \ell^{\prime}}>v_{j \ell}}\left[\frac{\gamma+\gamma^{\prime}}{2} \sin \left(\varphi_{j^{\prime} \ell^{\prime}}-\varphi_{j \ell}\right)\right. \\
& \left.+i \frac{\gamma-\gamma^{\prime}}{2} \cos \left(\varphi_{j^{\prime} \ell^{\prime}}-\varphi_{j \ell}\right)\right],
\end{aligned}
$$

and where $v_{j \ell}$ is the (previously introduced) discrete map returning the coupling point index for each pair $(j, \ell)$. Note that for isotropic coupling $\left(\gamma=\gamma^{\prime}\right)$, each $J_{j j^{\prime}}$ (for given $j$ and $j^{\prime}$ ) reduces to a sum of sin functions, where the argument of each sine is the phase shift associated with a pair of coupling points (one of atom $j$ one of $j^{\prime}$ ). Alternatively, $J_{j j^{\prime}}$ can be expressed by separating the right- and left-going contributions as

$$
J_{j j^{\prime}}=\gamma K_{j j^{\prime}}+\gamma^{\prime} K_{j j^{\prime}}^{*}
$$

with

$$
K_{j j^{\prime}}=\frac{1}{2} \sum_{v_{j^{\prime} \ell^{\prime}}>v_{j \ell}} e^{i\left(\varphi_{j \ell}-\varphi_{j^{\prime} \ell^{\prime}}+\frac{\pi}{2}\right)} .
$$

The issue is now raised as to whether or not $H_{\text {eff }} \neq 0$ when decoherence is inhibited [condition (26)]. It turns out that there generally exist patterns of coupling points such that $H_{\text {eff }}=0$ and patterns for which $H_{\text {eff }} \neq 0$, where the former yield a trivial dynamics (the system just does not evolve) and are thus unwanted. The best instance for illustrating this is a pair of giant atoms 1 and 2 , such that $\mathcal{N}_{1}=\mathcal{N}_{2}=2$, with equally spaced coupling points

$$
k_{0} x_{v}=(v-1) \varphi \quad \text { with } \quad v=1,2,3,4,
$$

the field being unidirectional $\left(\gamma^{\prime}=0\right)$. Three different types of patterns are then possible: serial, nested, and braided [see

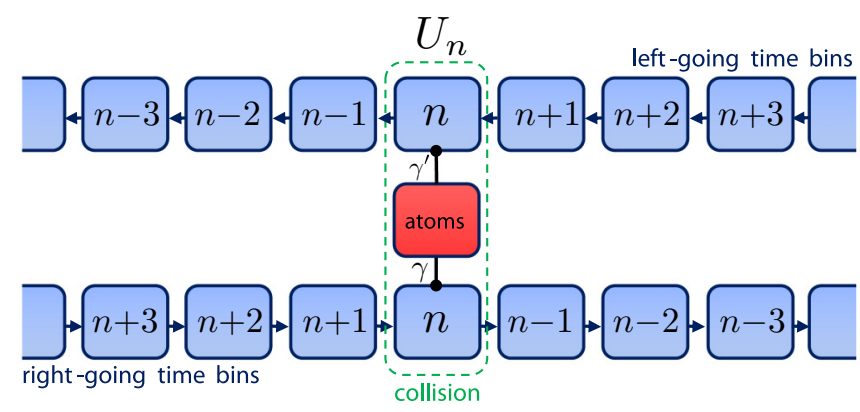

FIG. 5. Collisional picture of the joint dynamics of giant atoms and field. The field is decomposed into noninteracting time bins, each generally corresponding to the pair of harmonic oscillators $\left(b_{n}, b_{n}^{\prime}\right)$. At step $n$ (time interval $\left[t_{n-1}, t_{n}\right]$ ) the $n$th time bin collides with all the atoms according to coupling Hamiltonian $\bar{V}_{n}$ [cf. Eq. (33)]. The atoms at the same time are subject to an internal coherent dynamics described by Hamiltonian $\mathcal{H}_{n} \equiv H_{\text {eff }}$ [cf. Eq. (34)]. Both $\mathcal{H}_{n}$ and $\bar{V}_{n}$ are encompassed in the pairwise collision unitary $U_{n}$ [cf. Eqs. (4) and (5)]. In the unidirectional case $\gamma^{\prime}=0$, only right-going time bins $b_{n}$ are to be considered.

Figs. 4(a), 4(c) and 4(e)]. For the serial and nested topology, we choose $\varphi=\pi$, while in the braided case we take $\varphi=\pi / 2$. Each of these settings ensures that there is $(2 n+1) \pi$-phase shift between the two coupling points of each giant atom, thus matching the DF condition [recall Eq. (26)]. Using (38), in the serial and nested topologies we get $H_{\text {eff }}=0$, while the braided yields [14]

$$
H_{\mathrm{eff}}=\gamma\left(\sigma_{1} \sigma_{2}^{\dagger}+\sigma_{1}^{\dagger} \sigma_{2}\right)
$$

(we absorbed a phase factor $e^{-i \pi / 2}$ in the definition of $\sigma_{2}$ ). Analogous conclusions hold for isotropic coupling $\left(\gamma^{\prime}=\gamma=\right.$ $\Gamma / 2$ ), in which case (43) is generalized by replacing $\gamma$ with $\Gamma$.

Before concluding this section, we recall that, as discussed in Ref. [42], based on Eqs. (4), (5), (33), and (34) one can effectively see the joint dynamics as a collision model [43-47] (see Fig. 5). According to this (we first consider the unidirectional case $\gamma^{\prime}=0$ ), the field is decomposed into a discrete stream of right-going time bins, each being a bosonic mode with ladder operator $b_{n}$ defined by Eq. (35). During the time interval $\left[t_{n-1}, t_{n}\right]$, the $n$th time bin undergoes a collision with all the atoms at once, which is described by unitary $U_{n}$ [cf. Eq. (5)]. The atoms at the same time are effectively subject to a mutual coherent interaction described by $\mathcal{H}_{n} \equiv H_{\text {eff }}$ (encompassed in the collision unitary $U_{n}$ ). The extension to the bidirectional case is natural: there is now an additional stream of left-going time bins (each with ladder operator $b_{n}^{\prime}$ ). One can equivalently think of two-mode time bins $\left(b_{n}, b_{n}^{\prime}\right)$ such that in each collision the atoms collide with both time-bin subsystems $b_{n}$ and $b_{n}^{\prime}$ (see Fig. 5).

\section{MAPPING THE GIANT ATOMS DYNAMICS INTO A CASCADED COLLISION MODEL}

While, as pointed out in the previous section, the collision $U_{n}$ formally describes a simultaneous collision with all the atoms, we show next that it can be effectively decomposed as a cascade of subcollisions each involving only one coupling point. Cascaded collision models (for normal atoms) 
were introduced in Refs. [48,49] (see also Ref. [50]). Here a unidirectional waveguide is considered, the extension to the bidirectional case being postponed to Sec. IX.

For each coupling point $v$, let us define the interaction Hamiltonian

$$
V_{n v}=\sqrt{\frac{\gamma}{\Delta t}}\left(S_{v} b_{n}^{\dagger}+\text { H.c. }\right)
$$

coupling the $n$th time bin to atom $j$ with phase $\varphi_{j \ell}$ [cf. Eq. (27)], where $(j, \ell)$ is the pair corresponding to coupling point $v$ (in the remainder we introduce a convenient terminology and say that the time bin "interacts with the coupling point”). Using Eqs. (30), (31), (33), and (44), it is easily immediately checked that the average interaction Hamiltonian is just the sum of the $V_{n v}$ 's

$$
\bar{V}_{n}=\sum_{v} V_{n v}
$$

More importantly, as shown in the remainder, it turns out that, when the DF condition (26) is matched, the unitary collision $U_{n}$ can be decomposed as

$$
U_{n}=e^{-i V_{n \mathcal{N}} \Delta t} \cdots e^{-i V_{n 1} \Delta t} .
$$

Thereby, one can think of each collision (see Fig. 6) as the result of $\mathcal{N}$ cascaded subcollisions in each of which the time bin "collides" with one of the coupling points according to unitary $e^{-i V_{n v} \Delta t}$ with $V_{n v}$ given by (44). Of course this in particular entails that the same time bin collides with a given atom as many times as the number of respective coupling points $\mathcal{N}_{j}$. Yet the subcollisions with the same atom occur with different coupling Hamiltonians and are generally nonconsecutive (i.e., between two subcollisions with the same atom $j$ there may be subcollisions with atoms $j^{\prime} \neq j$ ), which is key to the occurrence of a nontrivial DF Hamiltonian as we will see shortly.

To prove (46), we expand to second order each subcollision unitary on the right-hand side as $e^{-i V_{n v} \Delta t} \simeq \mathbb{1}-i V_{n v} \Delta t-$ $\frac{1}{2} V_{n v}^{2} \Delta t^{2}$. This yields (to leading order)

$$
\prod_{\nu=1}^{\mathcal{N}} e^{-i V_{n v} \Delta t} \simeq \mathbb{1}-i\left(\bar{V}_{n}+\tilde{\mathcal{H}}_{n}\right) \Delta t-\frac{1}{2} \bar{V}_{n}^{2} \Delta t^{2},
$$

with the order in the product understood as in (46) and

$$
\tilde{\mathcal{H}}_{n}=i \frac{\Delta t}{2} \sum_{\nu>v^{\prime}}\left[V_{n v^{\prime}}, V_{n v}\right],
$$

where we used (45). Using (26), it is easily shown that $\tilde{\mathcal{H}}_{n}=$ $\mathcal{H}_{n} \equiv H_{\text {eff }}$ (see Appendix B). Upon comparison with (5), we thus conclude that (46) holds true.

The decomposition in terms of cascaded subcollisions in particular highlights the physical origin of effective Hamiltonian (34): if, instead of being sequential, the subcollisions occurred simultaneously (corresponding to perfectly colocated coupling points) then the overall collision unitary would be $e^{-i\left(\sum_{v} V_{n v}\right) \Delta t} \equiv e^{-i \bar{V}_{n} \Delta t}$, the corresponding secondorder expansion being just (47) without term $\tilde{\mathcal{H}}_{n} \equiv H_{\text {eff }}$. Thus the effective Hamiltonian arises precisely because the time bin collides with the coupling points in a cascaded fashion. This is in fact the same mechanism underpinning emergence of effective Hamiltonians in chiral quantum optics with normal

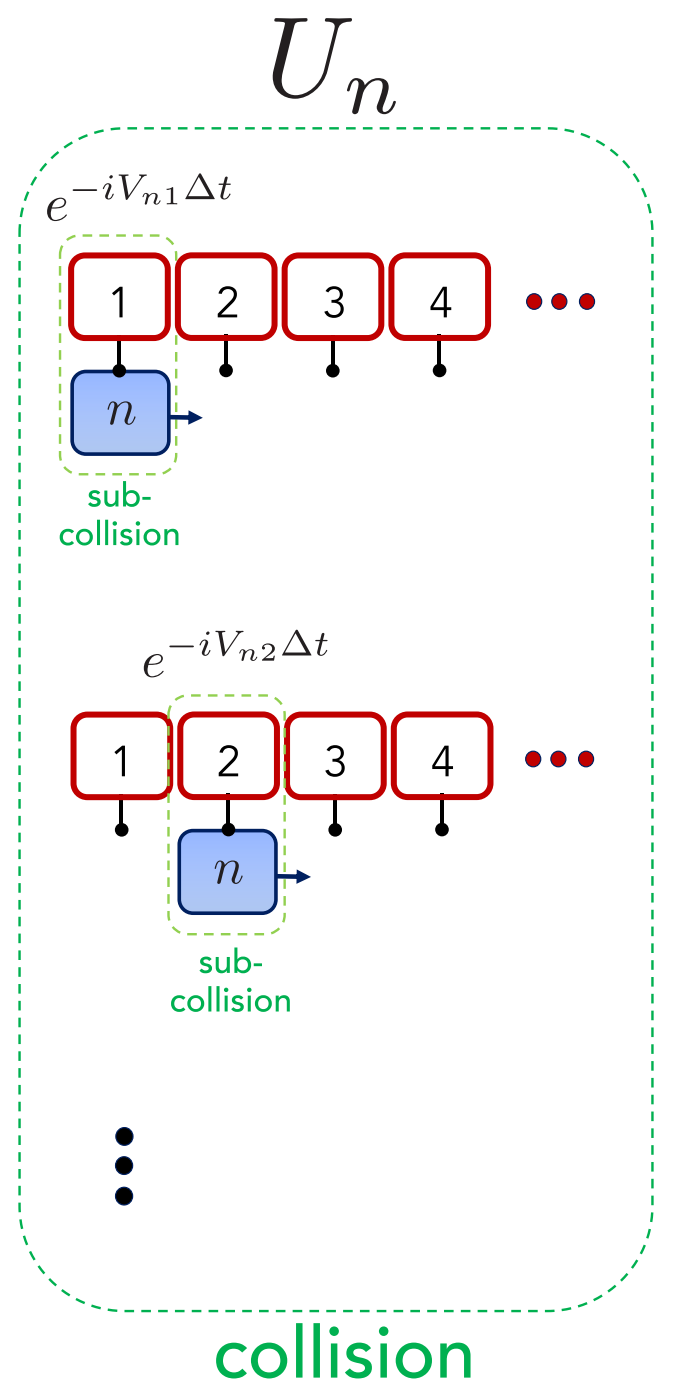

FIG. 6. Under the DF condition (26), each collision in Fig. 5 (we address here the case $\gamma^{\prime}=0$ ) can be effectively decomposed into $\mathcal{N}$ cascaded subcollisions according to Eq. (46). Each subcollision is between the same time bin $n$ and a different coupling point corresponding to coupling Hamiltonian (44) (time grows from top to bottom)

atoms [51], the difference yet being that decoherence cannot be suppressed in the latter case (because $\bar{V}_{n}$ cannot vanish with normal atoms).

\section{MECHANISM BEHIND EMERGENCE OF NONTRIVIAL $\boldsymbol{H}_{\text {eff }}$}

Occurrence of nontrivial (i.e., nonzero) DF Hamiltonians is simply interpreted in the cascaded-collision-model picture.

As in Sec. V A, throughout this and the next section we consider a unidirectional waveguide and giant atoms with two coupling points each, which captures most of the essential physics.

\section{A. Single giant atom}

Let us consider first a single giant atom and set $x_{1}=\tau_{1}=$ $0, \varphi=k_{0} x_{2}$. The DF condition $\bar{V}_{n}=0$ then simply reads 
$\varphi=(2 n+1) \pi$ with $n$ an integer number. Hence, $S_{1}=-S_{2}=$ $\sigma_{1}$ and [cf. Eq. (44)]

$$
V_{n 1}=\sqrt{\frac{\gamma}{\Delta t}}\left(\sigma_{1} b_{n}^{\dagger}+\text { H.c. }\right), \quad V_{n 2}=\sqrt{\frac{\gamma}{\Delta t}}\left(-\sigma_{1} b_{n}^{\dagger}+\text { H.c. }\right) \text {. }
$$

Thus $V_{n 1}=-V_{n 2}$ and [see Eq. (46)]

$$
U_{n}=e^{-i V_{n 2} \Delta t} e^{-i V_{n 1} \Delta t}=\mathbb{1},
$$

meaning that the collision has no effect overall. This, in particular, necessarily entails $H_{\text {eff }}=0$ [recall Eq. (5)]. In other words, the two subcollisions are the time reversed of one another (so that the net effect is null). To sum up, in order to ensure the DF condition $\bar{V}_{n}=0$ for a single giant atom, one must adjust the phase shift so that $V_{n 2}=-V_{n 1}$. This yet brings about that one subcollision is just the other one time reversed, trivially yielding $U_{n}=0$ hence $H_{\text {eff }}=0$.

\section{B. Two atoms}

When it comes to a pair of giant atoms, instead, conditions $\bar{V}_{n}=0$ and $U_{n} \neq 0$ can be matched simultaneously. To see this, we reconsider uniformly spaced atoms as in Eq. (42) and always set $\varphi$ so as to ensure a $(2 n+1) \pi$-phase shift between the pair of coupling points of each atom, hence $\bar{V}_{n}=0$ (similarly to the single-atom instance just discussed).

For convenience we define the coupling Hamiltonians

$$
\mathcal{V}_{j}=\sqrt{\frac{\gamma}{\Delta t}}\left(\sigma_{j} b_{n}^{\dagger}+\text { H.c. }\right)
$$

with $j=1,2$ (the dependence on $n$ is left implicit). No phase factor appears in this definition.

Consider first the serial scheme in Fig. 4(a), in which case we set $\varphi=\pi$. Then [see Fig. 4(b)]

$$
V_{n 1}=\mathcal{V}_{1}, \quad V_{n 2}=-\mathcal{V}_{1}, \quad V_{n 3}=\mathcal{V}_{2}, \quad V_{n 4}=-\mathcal{V}_{2}
$$

This results in the collision unitary [cf. (46)]

$$
U_{n}=e^{i \mathcal{V}_{2} \Delta t} e^{-i \mathcal{V}_{2} \Delta t} e^{i \mathcal{V}_{1} \Delta t} e^{-i \mathcal{V}_{1} \Delta t}=\mathbb{1},
$$

which is a trivial dynamics such that $H_{\mathrm{eff}}=0$. This case is in fact an extension of the single giant atom considered above.

For the nested case in Fig. 4(c), we set $\varphi=\pi$. Then [see Fig. 4(d)]

$$
V_{n 1}=\mathcal{V}_{1}, \quad V_{n 2}=-\mathcal{V}_{2}, \quad V_{n 3}=\mathcal{V}_{2}, \quad V_{n 4}=-\mathcal{V}_{1}
$$

Thus the second pair of subcollisions is the first pair timereversed

$$
U_{n}=e^{i \mathcal{V}_{1} \Delta t} e^{-i \mathcal{V}_{2} \Delta t} e^{i \mathcal{V}_{2} \Delta t} e^{-i \mathcal{V}_{1} \Delta t}=\mathbb{1},
$$

ensuing again a trivial dynamics and $H_{\mathrm{eff}}=0$. Equivalently, the pair of central subcollisions, both involving atom 2 , are the time reversed of one another. Thus atom 2 simply disappears from $U_{n}$, which reduces to $U_{n}=e^{i \mathcal{V}_{1} \Delta t} e^{-i \mathcal{V}_{1} \Delta t}=\mathbb{1}$.

For the braided arrangement of Fig. 4(e), we set $\varphi=\pi / 2$. Then [see Fig. 4(f)]

$$
V_{n 1}=\mathcal{V}_{1}, \quad V_{n 2}=\mathcal{V}_{2}, \quad V_{n 3}=-\mathcal{V}_{1}, \quad V_{n 4}=-\mathcal{V}_{2}
$$

[with $\mathcal{V}_{2}$ now defined by (51) for $j=2$ under the replacement $\left.\sigma_{2} \rightarrow-i \sigma_{2}\right]$. The collision unitary is given by

$$
U_{n}=e^{i \mathcal{V}_{2} \Delta t} e^{i \mathcal{V}_{1} \Delta t} e^{-i \mathcal{V}_{2} \Delta t} e^{-i \mathcal{V}_{1} \Delta t}=e^{-i \gamma\left(\sigma_{1} \sigma_{2}^{\dagger}+\sigma_{1}^{\dagger} \sigma_{2}\right) \Delta t} \neq \mathbb{1}
$$

Therefore, $\bar{V}_{n}=0$ is fulfilled but now $H_{\text {eff }} \neq 0$.

The above shows that, while being irrelevant for realizing the DF condition $\bar{V}_{n}=0$, the coupling points topology is crucial in order to have a nonvanishing effective Hamiltonian. In terms of propagators [cf. Eqs. (4) and (5)], this is ultimately due to the fact that the second-order term $\mathcal{H}_{n}$ is affected by the time-ordering operator, while $\bar{V}_{n}$ and (of course) $\bar{V}_{n}^{2}$ are fully insensitive to it.

\section{Many atoms}

The above arguments are naturally extended to more than two giant atoms. Again, the DF condition (26) is matched when for each atom the phase shift between its coupling points is a multiple integer of $\pi$, that is $\varphi_{j, 2}-\varphi_{j, 1}=\left(2 n_{j}+1\right) \pi$ for some integer $n_{j}$ and for any $j$. Based on the above discussion, we can state that if there exists an atom $j$ such that no coupling point of other atoms lies between its coupling points, i.e., $x_{j^{\prime}, \ell^{\prime}} \notin\left[x_{j, 1}, x_{j, 2}\right]$ for all $j^{\prime} \neq j$ and $\ell^{\prime}=1,2$, then $H_{\text {eff }}$ simply does not contain $\sigma_{j}$ and $\sigma_{j}^{\dagger}$ (i.e., atom $j$ is fully decoupled from the field and other atoms). This is because the pair of subcollision unitaries corresponding to coupling points $x_{j, 1}$ and $x_{j, 2}$ are the time reversed of one another, hence atom $j$ is fully decoupled from the field and all other atoms. A braided configuration is thus generally defined [14] as one such that no atom exhibits the above phenomenon.

\section{EQUIVALENT SCHEME USING A MEDIATOR}

Performing quantum information processing tasks [52] on two remote systems, say 1 and 2 , is a longstanding problem. The challenge is due to the impossibility of acting jointly on the systems (owing to their distance). A standard strategy to get around this is employing a mobile mediator: a third quantum system $M$ which can shuttle between 1 and 2 so as to mediate an indirect coupling, which, e.g., can be exploited for generating entanglement.

\section{A. Two-qubit gate}

The dynamics realizing the DF Hamiltonian for two giant atoms in a braided configuration [see Fig. 4(e)] in fact implements a two-qubit gate via a shuttling mediator. To see this, for simplicity and in view of the equivalent quantum circuit to be discussed later, we consider the field initially in the vacuum state, in which case each time bin behaves as an effective qubit $M$ [53]. Then $\mathcal{V}_{j}$ [cf. Eq. (51)] reads

$$
\mathcal{V}_{j}=\sqrt{\frac{\gamma}{\Delta t}}\left(\sigma_{j} \sigma_{M+}+\sigma_{j}^{\dagger} \sigma_{M-}\right)
$$

with $\sigma_{M-}=\sigma_{M+}^{\dagger}$ the usual spin-1/2 ladder operators of $M$. Note that, for convenience, we gauged away a phase factor $e^{-i \pi / 2}$ [due to $\varphi=\pi / 2$, cf. Eq. (42)] by absorbing it in the definition of $\sigma_{2}$.

Unitary (57) (braided configuration) can be interpreted as follows (see Fig. 7). $M$ is first put close to 1 and the $M-1$ 
(a) $e^{-i \mathcal{V}_{1} \Delta t}$

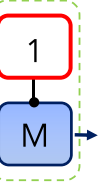

(c)

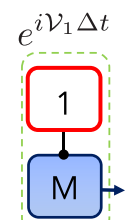

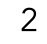

(b)
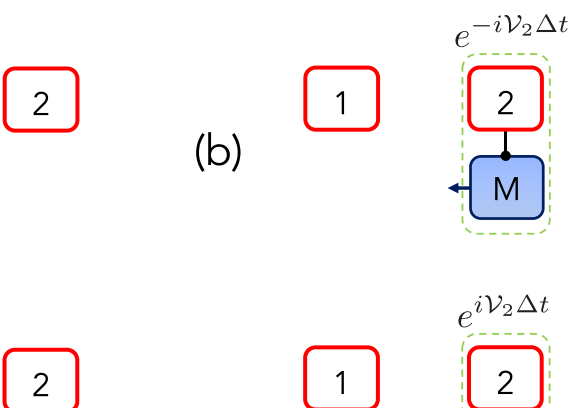

(d)

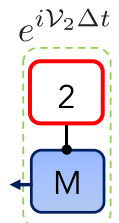

FIG. 7. The four subcollisions in the braided configuration can be seen as a mediator (embodied by the field time bin) shuttling between atoms 1 and 2 . This represents a collision model where in each collision the ancilla $M$ (time bin) interacts twice with each subsystem $j=1,2$ the first time with coupling Hamiltonian $\mathcal{V}_{j}$ [cf. Eq. (58)] and the second time with $-\mathcal{V}_{j}$. The net result is a coherent $1 \otimes 2$ interaction with $M$ eventually returning to its initial state. By applying phase kicks on $M$, one can make both subcollisions with $j$ occur with the same coupling $\mathcal{V}_{j}$ (see Fig. 8).

two-qubit gate $e^{-i \mathcal{V}_{1} \Delta t}$ applied on them [Fig. 7(a)], then $M$ is moved close to 2 and the $M-2$ gate $e^{-i \nu_{2} \Delta t}$ applied [Fig. 7(b)], next the $M$ goes back to 1 and gate $e^{i \mathcal{V}_{1} \Delta t}$ is applied [Fig. 7(c)]. Finally, $M$ lies close to 2 again and gate $e^{i \nu_{2} \Delta t}$ is applied [Fig. 7(d)].

Consider now the more realistic case that only one twoqubit gate per atom can be implemented, say $e^{-i \mathcal{V}_{1} \Delta t}$ and $e^{-i \mathcal{V}_{2} \Delta t}$ : we ask whether the other two can be obtained from these by adding extra single-qubit (local) gates. Noting that the local unitary transformation defined by $U_{M}=\sigma_{M z}$ transforms the $M$ 's ladder operators as $\sigma_{M \pm} \rightarrow-\sigma_{M \pm}$, we have

$$
\sigma_{M z} \mathcal{V}_{j} \sigma_{M z}=-\mathcal{V}_{j} \Rightarrow e^{i \mathcal{V}_{j} \Delta t}=\sigma_{M z} e^{-i \mathcal{V}_{j} \Delta t} \sigma_{M z},
$$

with $j=1$,2. Plugging the last decomposition of $e^{i \mathcal{V}_{j} \Delta t}$ in (57) thus yields

$$
U_{n}=\sigma_{M z} e^{-i \mathcal{V}_{2} \Delta t} e^{-i \mathcal{V}_{1} \Delta t} \sigma_{M z} e^{-i \mathcal{V}_{2} \Delta t} e^{-i \mathcal{V}_{1} \Delta t},
$$

where we used $\sigma_{M z}^{2}=1$. Recalling that in the braided configuration $U_{n}=e^{-i H_{\text {eff }} \Delta t}$ with $H_{\text {eff }}$ given by (43), we conclude

$$
e^{-i \gamma\left(\sigma_{1} \sigma_{2}^{\dagger}+\text { H.c. }\right) \Delta t}=\sigma_{M z} e^{-i \mathcal{V}_{2} \Delta t} e^{-i \mathcal{V}_{1} \Delta t} \sigma_{M z} e^{-i \mathcal{V}_{2} \Delta t} e^{-i \mathcal{V}_{1} \Delta t} .
$$

The collision unitary in the braided configuration-hence the entire dynamics in fact-can thus be seen as alternate subcollisions (of the same type) of $M$ with 1 and 2 where a local $\pi$-phase gate is applied on $M$ at the end of each cycle. This effectively implements a DF interaction between 1 and 2 only. Note that replacing $\sigma_{M z}=\operatorname{diag}\left(1, e^{i \pi}\right)$ (written in matrix form) with another phase gate $U_{M}=\operatorname{diag}\left(1, e^{i \varphi}\right)$ with $\varphi \neq$ $(2 n+1) \pi$ generally gives rise to an overall unitary $U_{n}$ which does not act trivially on $M$, thus introducing decoherence.

The above is somewhat reminiscent of dynamical decoupling schemes [54], where suitable local pulses are repeatedly applied in order to effectively decouple the system from the environment thus suppressing decoherence. Note however

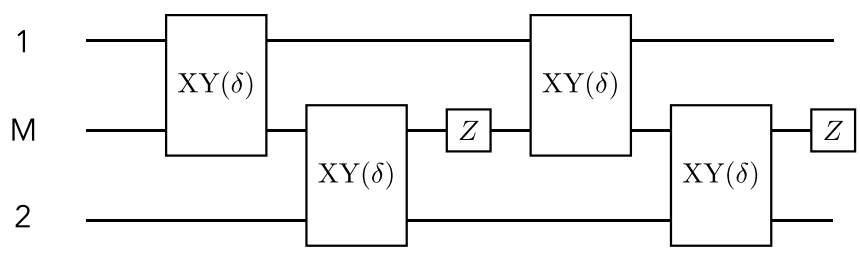

$$
\delta=\frac{1}{\pi} \sqrt{\gamma \Delta t}
$$

FIG. 8. Equivalent quantum circuit of the unitary collision in the braided scheme [cf. Eqs. (57) and (61)]. A (maximally entangling) i-SWAP two-qubit gate on 1 and 2 can be effectively obtained by iterating this quantum circuit.

that in our case the environment (embodied by $M$, that is the field in fact) has an active role since it allows 1 and 2 to effectively crosstalk.

\section{B. Quantum circuit}

To express (61) in the language of quantum circuits [52], we recall the definition of an XY gate, also known as piSWAP or parametric i-SWAP,

$$
\mathrm{XY}(\delta)=\left[\begin{array}{cccc}
1 & 0 & 0 & 0 \\
0 & \cos \pi \delta & -i \sin \pi \delta & 0 \\
0 & -i \sin \pi \delta & \cos \pi \delta & 0 \\
0 & 0 & 0 & 1
\end{array}\right],
$$

which coincides with each $M-j$ unitary $e^{-i \mathcal{V}_{j} \Delta t}$ for $\delta=$ $\frac{1}{\pi} \sqrt{\gamma \Delta t}$. Thereby we get the equivalent quantum circuit of the collision unitary (57) displayed in Fig. 8 (where $Z=\sigma_{z}$ ).

Thus, remarkably, the waveguide setup with giant atoms can be seen as implementing iterated applications of the elementary quantum circuit in Fig. 8. A canonical (maximally entangling) i-SWAP two-qubit gate $U_{n}=e^{-i\left(\sigma_{1} \sigma_{2}^{\dagger}+\text { H.c. }\right)}$ is obtained after $N=\left[(\gamma \Delta t)^{-1}\right]$ iterations [recall Eq. (32)].

\section{BIDIRECTIONAL CHIRAL WAVEGUIDE}

The considerations in Sec. VII naturally extend to a bidirectional waveguide. In Sec. VII A we saw that, in the unidirectional case, if an atom $j$ is such that $x_{j^{\prime}, \ell^{\prime}} \notin\left[x_{j, 1}, x_{j, 2}\right]$ for any $j^{\prime} \neq j$ and $\ell^{\prime}=1,2$ (configuration not braided), then $\sigma_{j}$ and $\sigma_{j}^{\dagger}$ do not appear in $H_{\text {eff }}$ under the DF condition. The same holds for a bidirectional waveguide since, according to Eqs. (38) and (40), if $J_{j j^{\prime}}$ vanishes in the unidirectional case $\gamma^{\prime}=0$, then so does in the bidirectional one (when $\gamma^{\prime} \neq 0$ ).

For completeness it is however worth showing that one can reach the same conclusion even through a purely collisional argument. To this aim, we note that, even for a bidirectional waveguide, under the DF condition (26) the collision unitary [cf. Eqs. (5), (33), and (34)] can be decomposed into cascaded subcollisions [cf. Eq. (46)] as

$$
U_{n}=e^{-i\left(V_{n \mathcal{N}}+V_{n 1}^{\prime}\right) \Delta t} \cdots e^{-i\left(V_{n 1}+V_{n \mathcal{N}}^{\prime}\right) \Delta t},
$$

with [cf. Eq. (44)]

$$
V_{n v}^{\prime}=\sqrt{\frac{\gamma^{\prime}}{\Delta t}}\left(S_{\nu}^{\prime} b_{n}^{\prime \dagger}+\text { H.c. }\right)
$$




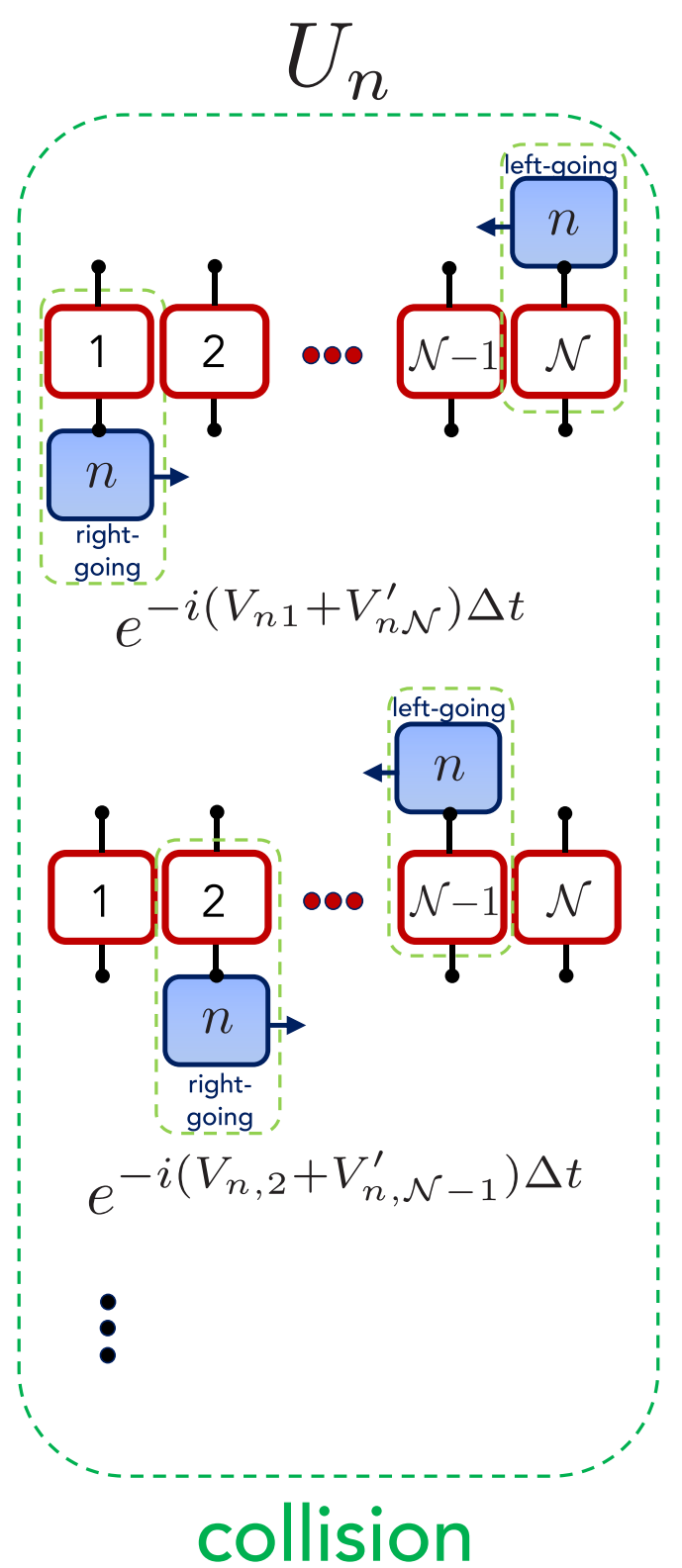

FIG. 9. Collision unitary $U_{n}$ 's decomposition into cascaded subcollisions for a bidirectional waveguide [cf. Eq. (63)]. In the vth subcollision, the right- and left-going time bins respectively interact with coupling points $v$ and $\mathcal{N}-v$ (time grows from top to bottom).

[recall Eqs. (27) and (35)]. This is because [cf. Eq. (5)] $\bar{V}_{n}=$ $\sum_{v}\left(V_{n v}+V_{n v}^{\prime}\right)$ and, as shown in Appendix $\mathrm{B}, \tilde{\mathcal{H}}_{n}=\mathcal{H}_{n} \equiv$ $H_{\text {eff }}$. Here $\tilde{\mathcal{H}}_{n}$ [cf. Eq. (48)] is now generally defined as

$$
\tilde{\mathcal{H}}_{n}=i \frac{\Delta t}{2} \sum_{\nu>v^{\prime}}\left[V_{n, v^{\prime}}+V_{n, \mathcal{N}+1-v^{\prime}}^{\prime}, V_{n, v}+V_{n, \mathcal{N}+1-v}^{\prime}\right] .
$$

Note that, as sketched in Fig. 9, in the first subcollision, the right-going time bin interacts with coupling point $v=1$ and the left-going time bin with coupling point $v=\mathcal{N}$. Then, in the second subcollision, the right- (left-)going time bin interacts with coupling point $v=2(v=\mathcal{N}-2)$ and so on.

In Appendix $C$ we use (63) to show that a giant atom does not appear in $H_{\text {eff }}$ whenever it is untangled from other atoms, i.e., when no coupling points of other atoms lie in between its two coupling points.

\section{MORE THAN TWO COUPLING POINTS}

The discussion in Sec. VII in many respects relied on the property that a single giant atom with a $\pi$-phase shift between its two coupling points (i.e., the DF condition) fully decouples from the field, i.e., $U_{n}=\mathbb{1}$ [cf. Eq. (50)]. For more than two coupling points, the DF condition for a single giant atom does not necessarily entail $U_{n}=\mathbb{1}$. The simplest example to see this is a single giant atom with three coupling points $(\mathcal{N} \equiv$ $\mathcal{N}_{1}=3$ ). The DF condition (26) occurs for (we drop subscript $j$ since there is only one atom; also we set $\varphi_{1}=0$ )

$$
\varphi_{2}=\frac{2 \pi}{3}+2 n \pi, \quad \varphi_{3}=\frac{4 \pi}{3}+2 m \pi,
$$

with $n, m$ integers. Plugging these into the effective Hamiltonian (38) for $\gamma^{\prime}=0$ we get

$$
H_{\text {eff }}=\frac{\gamma}{2}\left[2 \sin \left(\frac{2 \pi}{3}\right)+\sin \left(\frac{4 \pi}{3}\right)\right] \sigma_{z} \neq 0
$$

(the sum of the three sines is $\simeq 0.87$ ).

More generally, for an atom $j$ such that $x_{j^{\prime} \neq j, \ell^{\prime}} \notin$ $\left[x_{j, 1}, x_{j, \mathcal{N}_{j}}\right]$ and fulfilling the DF condition $\sum_{\ell} V_{n, v_{j, \ell}}=0$, in general

$$
e^{-i V_{n, v_{j, \mathcal{N}}} \Delta t} \cdots e^{-i V_{n, v_{j, 1}} \Delta t} \neq \mathbb{1},
$$

where $\mathcal{N}_{j}>2$ (if $\mathcal{N}_{j}=2$, the identity holds). However, (68) is anyway of the form $e^{-i \delta_{j} \sigma_{z} \Delta t}$ (with $\delta_{j}$ a frequency shift), hence all terms of $H_{\text {eff }}$ coupling $j$ to any other atom will vanish, i.e., in (38) $J_{j j^{\prime}} \neq 0$ only for $j^{\prime}=j$. Thus, if the only focus is coupling the atoms, then the braided topology remains the only one yielding a nontrivial $H_{\text {eff }}$. This remains true for a chiral waveguide $\left(\gamma^{\prime} \neq 0\right)$ since (40) shows that if $J_{j j^{\prime}}=0$ for $\gamma^{\prime}=0$, then it vanishes also for $\gamma^{\prime} \neq 0$.

\section{CONCLUSIONS}

In this work we investigated the physical mechanism underpinning implementation of DF Hamiltonians with giant atoms. We first introduced a general framework for obtaining DF Hamiltonians through second-order interactions mediated by an environment. The key "DF condition" is having an interaction Hamiltonian averaging to zero over a suitable coarse-grained timescale. The framework was first illustrated with standard dispersive Hamiltonians, in which case large detunings ensure a vanishing average interaction. We then considered giant atoms in a broadband waveguide and showed that, thanks to the nonlocal nature of the coupling, the DF condition can still be fulfilled, but in a qualitatively different way.

The above framework was then connected to a collisional picture of the joint dynamics of giant atoms and field in terms of elementary pairwise collisions between the atoms and field time bins. We showed that each collision can be decomposed as cascaded subcollisions, providing an intuitive understanding of the origin of the effective Hamiltonian. This was used to interpret the relationship between topology of the coupling points and occurrence of trivial/nontrivial DF Hamiltonians. 
In addition, we showed that the giant atoms dynamics can be mapped into a system shuttling between the atoms and subject to periodic phase kicks so as to effectively mediate a DF interatomic interaction, a mechanism in some respects reminiscent of dynamical decoupling schemes.

While here we did not consider lossy photonic environments $[19,36,55]$, the considered framework could be naturally extended to accommodate these. Likewise, a generalization to giant atoms in gapped structured reservoirs [56] appears viable.

On a methodological ground, we note that, although the collisional picture [42] and input-output/SLH formalism $[27,28]$ are equivalent descriptions (since the underlying microscopic model and approximations are identical), the aspects of giant-atoms dynamics which this work focused on are best tackled through the former approach. This is essentially due to the decomposition into two-body unitaries, the hallmark of the collisional description. In this respect, the present work in particular showcases a type of problem where this approach is particularly advantageous.

From the specific viewpoint of collision models and their quantum information processing applications (see, e.g., [57-59]), Secs. VI and VIII in fact introduce a class of cascaded collision models implementing maximally entangling multiqubit gates free from decoherence. Notably, these correspond to second-order effective Hamiltonians, at variance with schemes in Refs. [57,58] which are of first order (see also Ref. [45]).

\section{ACKNOWLEDGMENTS}

We would like to thank G. M. Palma, S. Lorenzo, G. Falci, and A. F. Kochum for fruitful discussions. We acknowledge support from MIUR through project PRIN Project 2017SRN-BRK QUSHIP. A.C. acknowledges support from the Government of the Russian Federation through Agreement No. 074-02-2018-330 (2).

\section{APPENDIX A: PROOF OF PROPERTY}

The propagator in each time interval (5) for $\bar{V}_{n}=0$ reduces to $U_{n}=\mathbb{1}-i \mathcal{H}_{n} \Delta t$. Accordingly,

$$
\sigma_{n}=\sigma_{n-1}-i\left[\mathcal{H}_{n}, \sigma_{n-1}\right] \Delta t .
$$

At the first step, $\sigma_{n-1}=\rho_{0} \otimes \rho_{E}$, thus using condition (11)

$$
\begin{aligned}
\sigma_{1} & =\rho_{0} \otimes \rho_{E}-i\left[\mathcal{H}_{n}, \rho_{0} \otimes \rho_{E}\right] \Delta t \\
& =\rho_{0} \otimes \rho_{E}-i\left[\mathcal{H}_{n}, \rho_{0} \otimes \mathbb{1}\right]\left(\mathbb{1} \otimes \rho_{E}\right) \Delta t \\
& =\left(\rho_{0}-i\left[H_{\mathrm{eff}}, \rho_{0}\right]\right) \otimes \rho_{E} \Delta t .
\end{aligned}
$$

This implies $\sigma_{1}=\rho_{1} \otimes \rho_{E}$ with

$$
\frac{\Delta \rho_{1}}{\Delta t}=-i\left[H_{\mathrm{eff}}, \rho_{0}\right]
$$

[recall Eq. (9)]. By induction, we get that at each step $\sigma_{n}=$ $\rho_{n} \otimes \rho_{E}$ with $\rho_{n}$ fulfilling

$$
\frac{\Delta \rho_{n}}{\Delta t}=-i\left[H_{\mathrm{eff}}, \rho_{n-1}\right]
$$

Taking the continuous-time limit, $\rho_{n-1} \rightarrow \rho_{t}, \Delta \rho_{n} / \Delta t \rightarrow \dot{\rho}$ so that (A4) reduces to Eq. (12).

\section{APPENDIX B: $\tilde{\mathcal{H}}_{n}=\mathcal{H}_{n}$}

Let us begin with the unidirectional case. Each commutator in (48) is explicitly worked out as

$\left[V_{n v^{\prime}}, V_{n v}\right]=\frac{\gamma}{\Delta t}\left(\left[S_{v^{\prime}}, S_{v}^{\dagger}\right]-\right.$ H.c. $) b_{n}^{\dagger} b_{n}+\frac{\gamma}{\Delta t}\left(S_{v^{\prime}}^{\dagger} S_{v}-\right.$ H.c. $)$.

Upon comparison with (34) for $\gamma^{\prime}=0$, the proof thus reduces to showing that the sum over $v>v^{\prime}$ of terms $\propto b_{n}^{\dagger} b_{n}$ vanish.

Each commutator $\left[S_{v^{\prime}}, S_{v}^{\dagger}\right]$ is nonzero only when coupling points $v$ and $v^{\prime}$ belong to the same atom. Thus, in light of (27),

$$
\begin{aligned}
\sum_{\nu>v^{\prime}}\left[S_{\nu}, S_{v^{\prime}}^{\dagger}\right]-\text { H.c. } & =\sum_{j} \sum_{\ell>\ell^{\prime}} e^{i\left(\varphi_{j \ell}-\varphi_{j \ell^{\prime}}\right)}\left[\sigma_{j}, \sigma_{j}^{\dagger}\right]-\text { H.c. } \\
& =\sum_{j}\left(\sum_{\ell>\ell^{\prime}} e^{i\left(\varphi_{j \ell}-\varphi_{j \ell^{\prime}}\right)}-\text { c.c. }\right) \sigma_{j z}
\end{aligned}
$$

(recall that $x_{j 1}<x_{j 2}<\cdots$ ). When (26) holds, the coefficient of $\sigma_{j z}$ vanishes for each $j$,

$$
\begin{aligned}
\sum_{\ell>\ell^{\prime}} e^{i\left(\varphi_{j \ell}-\varphi_{j \ell^{\prime}}\right)}-\text { c.c. } & =\sum_{\ell \geqslant \ell^{\prime}} e^{i\left(\varphi_{j \ell}-\varphi_{j \ell^{\prime}}\right)}-\text { c.c. } \\
& =\sum_{\ell=\ell^{\prime}}^{\mathcal{N}_{j}} e^{i \varphi_{j \ell}} \sum_{\ell^{\prime}=1}^{\mathcal{N}_{j}} e^{-i \varphi_{j \ell^{\prime}}}-\text { c.c. }=0 .
\end{aligned}
$$

Thus

$$
\sum_{\nu>v^{\prime}}\left[V_{n v^{\prime}}, V_{n v}\right]=\frac{\gamma}{\Delta t} \sum_{\nu>v^{\prime}}\left(S_{v^{\prime}}^{\dagger} S_{v}-\text { H.c. }\right),
$$

completing the proof.

In the bidirectional case, each commutator in (65) reads

$$
\begin{aligned}
& {\left[V_{n, v^{\prime}}+V_{n, \mathcal{N}+1-v^{\prime}}^{\prime}, V_{n, v}+V_{n, \mathcal{N}+1-v}^{\prime}\right]} \\
& =\left[V_{n, v^{\prime}}, V_{n, \nu}\right]+\left[V_{n, \mathcal{N}+1-v^{\prime}}^{\prime}, V_{n, \mathcal{N}+1-v}^{\prime}\right] \\
& \quad+\left[V_{n, \nu^{\prime}}, V_{n, \mathcal{N}+1-v}^{\prime}\right]+\left[V_{n, \mathcal{N}+1-v^{\prime}}^{\prime}, V_{n, \nu}\right] .
\end{aligned}
$$

The last line features terms $\propto\left[b_{n}^{\dagger}, b_{n}^{\prime}\right]$ and $\propto\left[b_{n}^{\prime \dagger}, b_{n}\right]$, which vanish because left- and right-going time-bin operators commute. Additionally, there are terms $\propto b_{n}^{\dagger} b_{n}^{\prime}$ (or $\propto b_{n}^{\prime \dagger} b_{n}$ ) featuring quantities like (B2) where however one of the two phases is primed: these vanish as well since (B3) holds even if $\varphi_{j \ell^{\prime}} \rightarrow \varphi_{j \ell^{\prime}}^{\prime}$. We are thus only left with terms analogous to (B1) given by

$$
\begin{aligned}
& {\left[V_{n, v^{\prime}}, V_{n, v}\right]+\left[V_{n, \mathcal{N}+1-v^{\prime}}^{\prime}, V_{n, \mathcal{N}+1-v}^{\prime}\right]} \\
& \quad=\frac{\gamma}{\Delta t}\left(S_{v^{\prime}}^{\dagger} S_{v}+S_{\mathcal{N}+1-v^{\prime}}^{\prime \dagger} S_{\mathcal{N}+1-v}^{\prime}-\text { H.c. }\right) .
\end{aligned}
$$

Summing this over $v>v^{\prime}$ yields $\frac{\gamma}{\Delta t}\left(S_{v^{\prime}}^{\dagger} S_{v}+S_{v^{\prime}}^{\dagger} S_{v^{\prime}}^{\prime}-\right.$ H.c. $)$ (where we used that $\mathcal{N}+1-v^{\prime}>\mathcal{N}+1-v$ for $v>v^{\prime}$ ), completing the proof.

\section{APPENDIX C: EXTENSION OF SEC. VIIA TO A BIDIRECTIONAL WAVEGUIDE}

In order to extend the considerations in Sec. VII A to a bidirectional waveguide, we essentially need to show that, for an atom $j$ such that $x_{j^{\prime}, \ell^{\prime}} \notin\left[x_{j, 1}, x_{j, 2}\right]$ for any $j^{\prime} \neq j$ and $\ell^{\prime}=1,2$ (recall Sec. VII A), $\sigma_{j}$ and $\sigma_{j}^{\dagger}$ do not appear in $H_{\text {eff }}$ under the DF condition. We first recall that $\bar{V}_{n}=0 \Leftrightarrow{\overline{V^{\prime}}}_{n}=0$ (since 
$S=0 \Leftrightarrow S^{\prime}=0$ ). In this configuration the overall coupling point index runs over $v=1,2, \ldots, k_{j}, k_{j}+1, \ldots, \mathcal{N}$ with $k_{j} \leftrightarrow(j, 1)$ and $k_{j}+1 \leftrightarrow(j, 2)$ labeling the left and right coupling points of atom $j$. Accordingly, in unitary (63), the subcollision unitaries involving the $j$ th atom are (to make notation lighter we drop subscript $n$ )

$$
\tilde{U}_{j}=e^{-i\left(V_{k_{j}+1}+V_{\mathcal{N}-k_{j}}^{\prime}\right) \Delta t} e^{-i\left(V_{k_{j}}+V_{\mathcal{N}+1-k_{j}}^{\prime}\right) \Delta t}
$$

and

$$
\tilde{W}_{j}=e^{-i\left(V_{\mathcal{N}+1-k_{j}}+V_{k_{j}}^{\prime}\right) \Delta t} e^{-i\left(V_{\mathcal{N}-k_{j}}+V_{k_{j}+1}^{\prime}\right) \Delta t} .
$$

Upon inspection, the pair of $V$ 's in each exponent commute because one involves a coupling point of atom $j$ and a rightgoing time bin, while the other one features a coupling point of an atom $j^{\prime} \neq j$ and a left-going time bin. Thereby, (C1) can be decomposed as

$$
\begin{aligned}
\tilde{U}_{j} & =e^{-i V_{k_{j}+1} \Delta t} e^{-i V_{\mathcal{N}-k_{j}}^{\prime} \Delta t} e^{-i V_{k_{j}} \Delta t} e^{-i V_{\mathcal{N}+1-k_{j}}^{\prime} \Delta t} \\
& =e^{-i V_{k_{j}+1} \Delta t} e^{-i V_{k_{j}} \Delta t} e^{-i V_{\mathcal{N}-k_{j}}^{\prime} \Delta t} e^{-i V_{\mathcal{N}+1-k_{j}}^{\prime} \Delta t} .
\end{aligned}
$$

Under the DF condition, $V_{k_{j}+1}=-V_{k_{j}}$ so that the first two exponentials in the last line reduce to the identity. An analogous conclusion holds for (C2). We thus conclude that $U_{n}$, hence $H_{\text {eff }}$, does not contain atom $j$, completing the proof.
[1] A. Sørensen and K. Mølmer, Quantum Computation with Ions in Thermal Motion, Phys. Rev. Lett. 82, 1971 (1999).

[2] A. Beige, D. Braun, B. Tregenna, and P. L. Knight, Quantum Computing Using Dissipation to Remain in a Decoherence-Free Subspace, Phys. Rev. Lett. 85, 1762 (2000).

[3] J. Kempe, D. Bacon, D. A. Lidar, and K. B. Whaley, Theory of decoherence-free fault-tolerant universal quantum computation, Phys. Rev. A 63, 042307 (2001).

[4] P. Facchi and S. Pascazio, Quantum zeno dynamics: Mathematical and physical aspects, J. Phys. A: Math. Theor. 41, 493001 (2008).

[5] E. Shahmoon and G. Kurizki, Nonradiative interaction and entanglement between distant atoms, Phys. Rev. A 87, 033831 (2013).

[6] J. S. Douglas, H. Habibian, C. L. Hung, A. V. Gorshkov, H. J. Kimble, and D. E. Chang, Quantum many-body models with cold atoms coupled to photonic crystals, Nat. Photonics 9, 326 (2015).

[7] V. Paulisch, H. J. Kimble, and A. González-Tudela, Universal quantum computation in waveguide QED using decoherence free subspaces, New J. Phys. 18, 043041 (2016).

[8] C. Cohen-Tannoudji, J. Dupont-Roc, G. Grynberg, and P. Thickstun, Atom-Photon Interactions: Basic Processes and Applications (Wiley Online Library, New York, 2004).

[9] H. P. Breuer, Foundations and measures of quantum nonMarkovianity, J. Phys. B: At. Mol. Opt. Phys. 45, 154001 (2012).

[10] S.-B. Zheng and G.-C. Guo, Efficient Scheme for Two-Atom Entanglement and Quantum Information Processing in Cavity Qed, Phys. Rev. Lett. 85, 2392 (2000).

[11] J. Majer, J. M. Chow, J. M. Gambetta, J. Koch, B. R. Johnson, J. A. Schreier, L. Frunzio, D. I. Schuster, A. A. Houck, A. Wallraff, A. Blais, M. H. Devoret, S. M. Girvin, and R. J. Schoelkopf, Coupling superconducting qubits via a cavity bus, Nature (London) 449, 443 (2007).

[12] C. D. Ogden, E. K. Irish, and M. S. Kim, Dynamics in a coupled-cavity array, Phys. Rev. A 78, 063805 (2008).

[13] D. E. Chang, J. S. Douglas, A. González-Tudela, C.-L. Hung, and H. J. Kimble, Colloquium: Quantum matter built from nanoscopic lattices of atoms and photons, Rev. Mod. Phys. 90, 031002 (2018).
[14] A. Frisk Kockum, G. Johansson, and F. Nori, Decoherence-Free Interaction Between Giant Atoms in Waveguide Quantum Electrodynamics, Phys. Rev. Lett. 120, 140404 (2018).

[15] B. Kannan, M. Ruckriegel, D. Campbell, A. Frisk Kockum, J. Braumüller, D. Kim, M. Kjaergaard, P. Krantz, A. Melville, B. M. Niedzielski et al., Waveguide quantum electrodynamics with giant superconducting artificial atoms, Nature (London) 583, 775 (2020).

[16] A. Frisk Kockum, Quantum optics with giant atoms - the first five years, Int. Symp. on Math., Quan. Theo., and Cryptog. 33, 125 (2020).

[17] A. Frisk Kockum, P. Delsing, and G. Johansson, Designing frequency-dependent relaxation rates and Lamb shifts for a giant artificial atom, Phys. Rev. A 90, 013837 (2014).

[18] L. Guo, A. Grimsmo, A. Frisk Kockum, M. Pletyukhov, and G. Johansson, Giant acoustic atom: A single quantum system with a deterministic time delay, Phys. Rev. A 95, 053821 (2017).

[19] T. M. Karg, B. Gouraud, P. Treutlein, and K. Hammerer, Remote Hamiltonian interactions mediated by light, Phys. Rev. A 99, 063829 (2019).

[20] G. Andersson, B. Suri, L. Guo, T. Aref, and P. Delsing, Nonexponential decay of a giant artificial atom, Nat. Phys. 15, 1123 (2019).

[21] P.-O. Guimond, B. Vermersch, M. L. Juan, A. Sharafiev, G. Kirchmair, and P. Zoller, A unidirectional on-chip photonic interface for superconducting circuits, npj Quantum Inform. 6, 1 (2020).

[22] L. Guo, A. Frisk Kockum, F. Marquardt, and G. Johansson, Oscillating bound states for a giant atom, Phys. Rev. Research 2, 043014 (2020).

[23] A. M. Vadiraj, A. Ask, T. G. McConkey, I. Nsanzineza, C. W. Sandbo Chang, A. Frisk Kockum, and C. M. Wilson, Engineering the level structure of a giant artificial atom in waveguide quantum electrodynamics, arXiv:2003.14167.

[24] D. Witthaut and A. S. Sorensen, Photon scattering by a threelevel emitter in a one-dimensional waveguide, New J. Phys. 12, 043052 (2010).

[25] Y. L. L. Fang, F. Ciccarello, and H. U. Baranger, NonMarkovian dynamics of a qubit due to single-photon scattering in a waveguide, New J. Phys. 20, 043035 (2018).

[26] I. C. Hoi, A. Frisk Kockum, L. Tornberg, A. Pourkabirian, G. Johansson, P. Delsing, and C. M. Wilson, Probing the quantum 
vacuum with an artificial atom in front of a mirror, Nat. Phys. 11, 1045 (2015).

[27] J. Combes, J. Kerckhoff, and M. Sarovar, The SLH framework for modeling quantum input-output networks, Adv. Phys.: X 2, 784 (2017).

[28] K. Lalumière, B. C. Sanders, A. F. Van Loo, A. Fedorov, A. Wallraff, and A. Blais, Input-output theory for waveguide QED with an ensemble of inhomogeneous atoms, Phys. Rev. A 88, 043806 (2013).

[29] R. R. Ernst, G. Bodenhausen, A. Wokaun et al., Principles of Nuclear Magnetic Resonance in One and Two Dimensions (Clarendon, Oxford, 1987), Vol. 14.

[30] L. M. K. Vandersypen and I. L. Chuang, NMR techniques for quantum control and computation, Rev. Mod. Phys. 76, 1037 (2005).

[31] D. F. James and J. Jerke, Effective Hamiltonian theory and its applications in quantum information, Can. J. Phys. 85, 625 (2007).

[32] O. Gamel and D. F. V. James, Time-averaged quantum dynamics and the validity of the effective Hamiltonian model, Phys. Rev. A 82, 052106 (2010).

[33] P. G. Di Stefano, E. Paladino, T. J. Pope, and G. Falci, Coherent manipulation of noise-protected superconducting artificial atoms in the lambda scheme, Phys. Rev. A 93, 051801(R) (2016).

[34] W. Magnus, On the exponential solution of differential equations for a linear operator, Commun. Pure Appl. Math. 7, 649 (1954).

[35] S. Haroche and J.-M. Raimond, Exploring the Quantum: Atoms, Cavities, and Photons (Oxford University Press, Oxford, 2006).

[36] F. Reiter and A. S. Sørensen, Effective operator formalism for open quantum systems, Phys. Rev. A 85, 032111 (2012).

[37] L. Peters, Young Scientists Journal (Oxford University Press, Oxford, UK, 2012), Vol. 5, p. 11.

[38] D. Roy, C. M. Wilson, and O. Firstenberg, Colloquium: Strongly interacting photons in one-dimensional continuum, Rev. Mod. Phys. 89, 021001 (2017).

[39] Z. Liao, X. Zeng, H. Nha, and M. S. Zubairy, Photon transport in a one-dimensional nanophotonic waveguide QED system, Phys. Scr. 91, 063004 (2016).

[40] X. Gu, A. Frisk Kockum, A. Miranowicz, Y. xi Liu, and F. Nori, Microwave photonics with superconducting quantum circuits, Phys. Rep. 718-719, 1 (2017).

[41] So long as we deal with giant atoms having two coupling points each, a trivial Hamiltonian is always identically zero. Later on, in Sec. X, we will see that a $H_{\text {eff }}$ can be nonzero but feature zero inter-atomic couplings, this being still trivial when the goal is coupling the atoms.

[42] D. Cilluffo, A. Carollo, S. Lorenzo, J. A. Gross, G. M. Palma, and F. Ciccarello, Collisional picture of quantum optics with giant emitters, Phys. Rev. Research 2, 043070 (2020).

[43] F. Ciccarello, Collision models in quantum optics, Quantum Meas. Quantum Metrol. 4, 53 (2017).

[44] T. A. Brun, A simple model of quantum trajectories, Am. J. Phys. 70, 719 (2002).

[45] N. Altamirano, P. Corona-Ugalde, R. B. Mann, and M. Zych, Unitarity, feedback, interactions-Dynamics emergent from repeated measurements, New J. Phys. 19, 013035 (2017).

[46] S. Lorenzo, F. Ciccarello, and G. M. Palma, Composite quantum collision models, Phys. Rev. A 96, 032107 (2017).

[47] J. A. Gross, C. M. Caves, G. J. Milburn, and J. Combes, Qubit models of weak continuous measurements: Markovian conditional and open-system dynamics, Quantum Sci. Technol. 3, 024005 (2018).

[48] V. Giovannetti and G. M. Palma, Master Equations for Correlated Quantum Channels, Phys. Rev. Lett. 108, 040401 (2012).

[49] V. Giovannetti and G. M. Palma, Master equation for cascade quantum channels: A collisional approach, J. Phys. B: At. Mol. Opt. Phys. 45, 154003 (2012).

[50] S. Lorenzo, A. Farace, F. Ciccarello, G. M. Palma, and V. Giovannetti, Heat flux and quantum correlations in dissipative cascaded systems, Phys. Rev. A 91, 022121 (2015).

[51] P. Lodahl, S. Mahmoodian, S. Stobbe, A. Rauschenbeutel, P. Schneeweiss, J. Volz, H. Pichler, and P. Zoller, Chiral quantum optics, Nature (London) 541, 473 (2017).

[52] Y. Wang, Statistical Science (Cambridge University Express, Cambridge, 2012), Vol. 27, pp. 373-394.

[53] In this case, before the collision, the time bin is in the vacuum state $\varrho_{n}=|0\rangle_{n}\langle 0|$. Using (44) and (46), one can check that in $U_{n} \varrho_{n}|0\rangle_{n}\langle 0| U_{n}^{\dagger}$ terms $\sim\left|k^{\prime}\right\rangle_{n}\langle k|$ with $k\left(\right.$ or $\left.k^{\prime}\right)=2,3, \ldots$ are at least of order $\sim \Delta t^{3 / 2}$. Thus the time bin behaves as a qubit with computational basis $\left\{|0\rangle_{n},|1\rangle_{n}\right\}$.

[54] L. Viola, E. Knill, and S. Lloyd, Dynamical Decoupling of Open Quantum Systems, Phys. Rev. Lett. 82, 2417 (1999).

[55] A. Sondberg Sørensen and K. Mølmer, Entangling atoms in bad cavities, Phys. Rev. A 66, 022314 (2002).

[56] A. González-Tudela, C. S. Muñoz, and J. I. Cirac, Engineering and Harnessing Giant Atoms in High-Dimensional Baths: A Proposal for Implementation with Cold Atoms, Phys. Rev. Lett. 122, 203603 (2019).

[57] B. Sutton and S. Datta, Manipulating quantum information with spin torque, Sci. Rep. 5, 17912 (2015).

[58] D. Layden, E. Martín-Martínez, and A. Kempf, Universal scheme for indirect quantum control, Phys. Rev. A 93 040301(R) (2016).

[59] B. Çakmak, S. Campbell, B. Vacchini, Ö. E. Müstecaplıŏglu, and M. Paternostro, Robust multipartite entanglement generation via a collision model, Phys. Rev. A 99, 012319 (2019). 